\title{
AN ALGORITHM TO COMPUTE THE TRANSITIVE CLOSURE, A TRANSITIVE APPROXIMATION AND A TRANSITIVE OPENING OF A PROXIMITY*
}

\author{
LUIS GARMENDIA \\ Universidad Complutense of Madrid, Spain \\ lgarmend@fdi.ucm.es \\ RAMÓN GONZALEZ \\ Universidad Complutense of Madrid, Spain \\ rgonzle@estad.ucm.es \\ JORDI RECASENS \\ Universitat Politècnica de Catalunya, Spain \\ j.recasens@upc.edu
}

\begin{abstract}
A method to get the transitive closure, a transitive opening and a transitive approximation of a reflexive and symmetric fuzzy relation is presented. The method builds at the same time a binary partition tree for the output similarities.
\end{abstract}

\section{Introduction}

Equivalence relations are important in many branches of knowledge and especially in Classification theories and Cluster Analysis since they generate a partition on the universe of discourse and permit to classify their elements and make clusters. In many cases the relation we start with is not an equivalence relation but only a reflexive and symmetric one.

A very important family of fuzzy relations are T-indistinguishabilities (reflexive, symmetric and T-transitive fuzzy relations) since they generalize (fuzzify) the concepts of (crisp) equivalence relation and equality [Trillas and Valverde 1984] and are useful to represent the ideas of similarity and neighbourhood as well.

Among T-indistinguishabilities, the ones which are transitive with respect to the Minimum t-norm are called similarities and are especially interesting and widely used in Taxonomy since they generate indexed hierarchical trees.

How to obtain T-indistinguishabilities and especially similarities from a given proximity relation $\mathrm{R}$, has become a very important task and there are many algorithms to do it. Many of them calculate the smallest T-indistinguishability

\footnotetext{
* Research partially supported by DGICYT projects number TIN2006-14311 and TIN2006-06190.
} 
greater of equal than $\mathrm{R}$, which is called its T-transitive closure. Other methods calculate $\mathrm{T}$-transitive openings, which are $\mathrm{T}$-indistinguishabilities smaller that $\mathrm{R}$ but maximal among all T-indistinguishabilities smaller than $\mathrm{R}$. Though the $\mathrm{T}$ transitive closure of a fuzzy proximity is unique, it is not the case of the Ttransitive openings and though there are some algorithms to calculate some of them, there is still a very interesting open problem to find all T-transitive openings of a given fuzzy proximity. Less attention has been paid to the obtention of T-indistinguishability operators not comparable with $\mathrm{R}$ in the sense that some of the entries are greater while some smaller than the corresponding entries of R. These T-transitive relations will be called T-transitive approximations of $\mathrm{R}$ in this paper. Despite the little interest since now in these approximations, it is obvious their importance since if we must replace a given fuzzy proximity by a T-transitive one, it is clear that in most occasions they will be closer to our relation than its corresponding T-transitive closure or some of its T-transitive openings ([Garmendia and Recasens 2007]).

This paper provides a simple algorithm to produce similarities (and therefore indexed hierarchical trees) from a fuzzy proximity relation R. Several forms of defining weights allow to easily computing the transitive closure of $\mathrm{R}$, a transitive opening and different transitive approximations of R. An interesting feature of it is that with the same algorithm the T-transitive closure, a Ttransitive opening and several $\mathrm{T}$-transitive approximations of $\mathrm{R}$ are generated.

\section{Preliminaries}

This section contains some definitions and properties of similarities and Tindistinguishabilities and some methods to build them from fuzzy proximity relations.

Definition 1: Let $E=\left\{e_{1}, \ldots, e_{n}\right\}$ be a finite set. A fuzzy relation $R$ on $E$ is a map R: $E \times E \rightarrow[0,1]$. The relation degree value for elements $e_{i}$ and $e_{j}$ in $E$ is called $\mathrm{e}_{\mathrm{ij} .}$. So $\mathrm{e}_{\mathrm{ij}}=\mathrm{R}\left(\mathrm{e}_{\mathrm{i}}, \mathrm{e}_{\mathrm{j}}\right)$.

A fuzzy relation $R$ is reflexive if $e_{i i}=1$ for all $1 \leq \mathrm{i} \leq \mathrm{n}$.

A fuzzy relation $\mathrm{R}$ is $\alpha$-reflexive if $\mathrm{e}_{\mathrm{ii}} \geq \alpha$ for all $1 \leq \mathrm{i} \leq \mathrm{n}$.

The relation $R$ is symmetric if $e_{i j}=e_{j i}$ for all $1 \leq i, j \leq n$.

A reflexive and symmetric fuzzy relation is called a fuzzy proximity relation.

Definition 2. Let T be a triangular norm [Schweizer, Sklar; 1984]. A fuzzy relation $\mathrm{R}: \mathrm{E} \times \mathrm{E} \rightarrow[0,1]$ is T-transitive if and only if $\mathrm{T}(\mathrm{R}(\mathrm{a}, \mathrm{b}), \mathrm{R}(\mathrm{b}, \mathrm{c})) \leq \mathrm{R}(\mathrm{a}$, c) for all a, b, c in E. In a fuzzy logic context it can be interpreted as 'The sentence "If a is related to b and b is related to c, then a is related to c" is true'.

Definition 4. [Zadeh 1971] A fuzzy similarity is a reflexive, symmetric and min-transitive fuzzy relation.

The T-transitive closure of a symmetric fuzzy relation is also symmetric. Also reflexivity and $\alpha$-reflexivity are preserved by the T-transitive closure. 


\section{Algorithm to compute the transitive closure, a transitive opening and a transitive approximation of a fuzzy proximity generating a binary partition tree.}

This section presents an algorithm that allows the computation of the transitive closure, a transitive opening and several other approximations of a give fuzzy proximity R. The fact that the same algorithm generates all these kind of approximations of R simplifies calculations and makes it a good tool to solve the problem of approximating fuzzy proximities, since the user can choose which kind of T-transitive approximation wants or needs.

Lemma 2. [Lee 2001] Let C and D be two fuzzy relations and $\mathrm{E}(\mathrm{f} ; \mathrm{C}, \mathrm{D})=\left(\begin{array}{ll}\hline C & F^{T} \\ F & D\end{array}\right)$ where all values in the box $\mathrm{F}$ are $\mathrm{f}$.

If $\mathrm{C}$ and $\mathrm{D}$ are fuzzy similarities, then $\mathrm{E}(\mathrm{f} ; \mathrm{C}, \mathrm{D})=\mathrm{E}$ is also a fuzzy similarity, $\forall \mathrm{f} \in[0, \min (\min (\mathrm{C}), \min (\mathrm{D}))]$.

The algorithm goes as follows.

Algorithm 1. Let $R$ be a fuzzy proximity relation on a universe $E=\left\{e_{1}, \ldots, e_{n}\right\}$ with values $e_{i j}=R\left(e_{i}, e_{j}\right)$. Lets call node to a subset of $E$ (a node is an element of $\wp(\mathrm{E})$ ). In order to make an easier notation, we consider the elements of $\mathrm{E}$ by their natural number of their position.

Input: a proximity $\mathrm{R}$

Output: Partition tree T (and matrix) of the transitive closure A = [aij], a transitive opening $\mathrm{B}=$ [bij] and a transitive approximation $\mathrm{C}=$ [cij] from $\mathrm{R}$. The given algorithm is the following:

1) Create a set of nodes $N$ initially with a set of singletons $\mathrm{N}_{i}=\left\{e_{i}\right\}$ for each element $e_{i}$ in $E$.

2) Set $a_{i i}=1, b_{i i}=1$, and $c_{i i}=1$ for all $i$ from 1 to $n$.

3) n-1 times (while $N$ is not the universe $E$ ) \{

Compute $\mathbf{m}\left(\mathbf{N}_{\mathbf{i}}, \mathbf{N}_{\mathbf{j}}\right)=\max _{i \in N_{i} j \in N_{j}} e_{i, j}$ for all pair of nodes $\mathbf{N} \times \mathbf{N}$ with $\mathbf{i \neq j}$.

Record $(i, j)$ where $m\left(N_{i}, N_{j}\right)$ is maximal.

Assign $\mathbf{a}_{\mathbf{r s}}=\mathbf{a}_{\mathbf{s r}}:=\max _{i \in N_{i} j \in N_{j}} e_{i, j}$ for all $\mathbf{r} \in \mathbf{N}_{\mathbf{i}}$ and $\mathbf{s} \in \mathbf{N}_{\mathbf{j}}$.

Assign $\mathbf{b}_{\mathbf{r s}}=\mathbf{b}_{\mathrm{sr}}:=\min \left(\min _{i \in N_{i} j \in N_{j}} e_{i, j}, \min _{k, l \in N_{i}} \mathrm{~b}_{k, l}, \min _{k, l \in N_{j i}} \mathrm{~b}_{k, l}\right)$ for all $r \in N_{i}$ and $s \in N_{j}$. 


$$
\text { Assign } \mathbf{c}_{\mathrm{rs}}=\mathbf{c}_{\mathrm{sr}}:=\min \left(\underset{i \in N_{i}}{\operatorname{avg} j \in N_{j}} e_{i, j}, \min _{k, l \in N_{i}} \mathrm{c}_{k, l}, \min _{k, l \in N_{j i}} \mathrm{c}_{k, l}\right) \text { for }
$$

all $r \in N_{i}$ and $s \in N_{j}$.

Delete nodes $\mathrm{N}_{\mathrm{i}}$ and $\mathrm{N}_{\mathrm{j}}$ from $\mathrm{N}$.

Insert $\mathbf{N}_{\mathbf{i}} \cup \mathbf{N}_{\mathbf{j}}$ into $\mathbf{N}$.

\}

avg is an idempotent aggregation operator. In particular, avg can be any quasiarithmetic mean such the arithmetic or geometric means, or an OWA operator, including the Minimum (corresponding to a T-transitive opening). Using different aggregator operators in this algorithm can provide different transitive approximations (or even a set of them computing several similarities $\mathrm{Bi}$ ) taking the same time complexity.

The trees generated by A, B and C coincide. Only their weights differ.

The algorithm takes just $n-1$ steps, where $n$ is the cardinality of the universe $E$. It takes $\mathrm{O}(\mathrm{n} 2)$ space complexity and $\mathrm{O}(\mathrm{n} 2 \log \mathrm{n})$ average time complexity.

\subsection{Example :}

Let $\mathrm{R}$ be the fuzzy proximity given by the following matrix:

$$
\mathrm{R}=\left(\begin{array}{cccccc}
1 & 1 & 0.5 & 0.3 & 0.2 & 0.3 \\
1 & 1 & 0.8 & 0.2 & 0.4 & 0.3 \\
0.5 & 0.8 & 1 & 0.9 & 0.3 & 0.3 \\
0.3 & 0.2 & 0.9 & 1 & 0.8 & 0.1 \\
0.2 & 0.3 & 0.3 & 0.8 & 1 & 0.5 \\
0.3 & 0.2 & 0.3 & 0.1 & 0.5 & 1
\end{array}\right)
$$

The first two loops of the part 3) of the algorithm records $m(N 1, N 2)=1$ and $\mathrm{m}(\mathrm{N} 3, \mathrm{~N} 4)=0,9$.

In the third loop a maximal value it is found with $m\left(\mathrm{~N}_{3} \cup \mathrm{N}_{4}, \mathrm{~N}_{5}\right)=0.8$ The matrix construction of the transitive closure $\mathrm{A}$, transitive opening $\mathrm{B}$ and transitive approximation $\mathrm{C}$ is in this step as follows

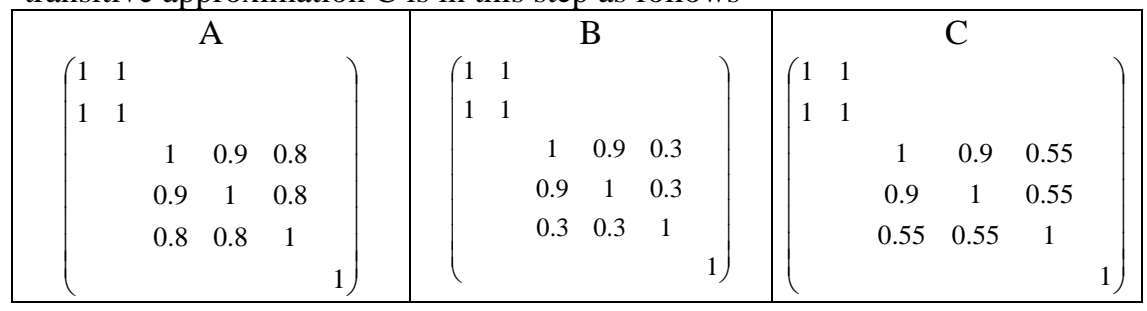

Figure 1 Transitive closure A, transitive opening B and transitive approximation $\mathrm{C}$ in step 3. In one more step, $\mathrm{N}_{1} \cup \mathrm{N}_{2}$ and $\mathrm{N}_{3}$ 
The matrix construction of the transitive closure A, transitive opening B and transitive approximation $\mathrm{C}$ is in this step as follows.

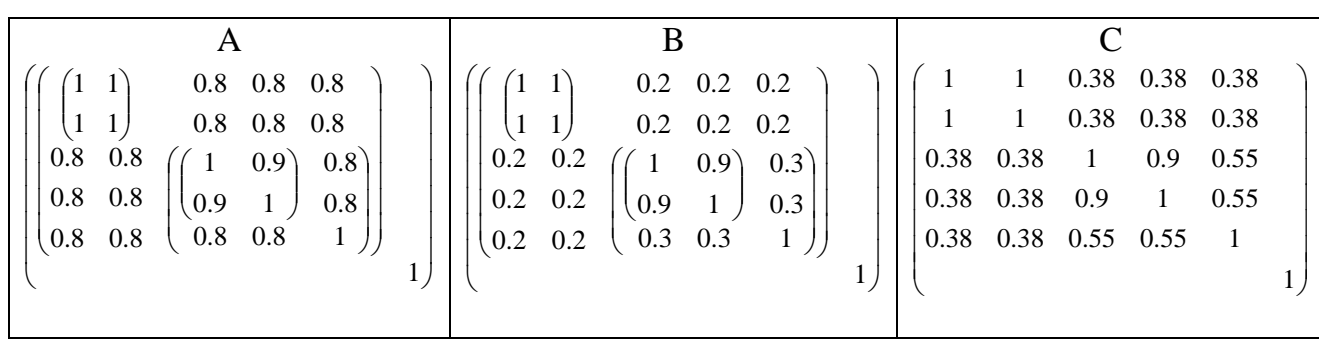

Figure 2 Transitive closure A, transitive opening B and transitive approximation C is in loop 4.

Finally, the last node is linked in loop 5. Note that there are only five (n-1) loops because the universe $\mathrm{E}$ has 6 elements.

\begin{tabular}{|c|c|c|c|c|c|c|c|c|c|c|c|c|c|c|c|c|}
\hline \multicolumn{6}{|c|}{ A } & \multicolumn{5}{|c|}{ B } & \multicolumn{6}{|c|}{ C } \\
\hline 1 & 1 & 0.8 & 0.8 & 0.8 & 0.5 & $\int\left(\left(\begin{array}{ll}1 & 1\end{array}\right)\right.$ & 0.2 & 0.2 & 0.2 & $0.1)$ & 1 & 1 & 0.38 & 0.38 & 0.38 & $0.28)$ \\
\hline 1 & 1 & 0.8 & 0.8 & 0.8 & 0.5 & $\left(\begin{array}{ll}1 & 1\end{array}\right)$ & 0.2 & 0.2 & 0.2 & 0.1 & 1 & 1 & 0.38 & 0.38 & 0.38 & 0.28 \\
\hline 0.8 & 0.8 & 1 & 0.9 & 0.8 & 0.5 & $0.2 \quad 0.2$ & 1 & $0.9)$ & $0.3)$ & 0.1 & 0.38 & 0.38 & 1 & 0.9 & 0.55 & 0.28 \\
\hline 0.8 & 0.8 & 0.9 & 1 & 0.8 & 0.5 & $0.2 \quad 0.2$ & 0.9 & 1 & 0.3 & 0.1 & 0.38 & 0.38 & 0.9 & 1 & 0.55 & 0.28 \\
\hline 0.8 & 0.8 & 0.8 & 0.8 & 1 & 0.5 & $\begin{array}{ll}0.2 & 0.2\end{array}$ & 0.3 & 0.3 & $\left.1 \int\right)$ & 0.1 & 0.38 & 0.38 & 0.55 & 0.55 & 1 & 0.28 \\
\hline 0.5 & 0.5 & 0.5 & 0.5 & 0.5 & 1 & $\begin{array}{lll} & 0.1 & 0.1\end{array}$ & 0.1 & 0.1 & 0.1 & $1)$ & 0.28 & 0.28 & 0.28 & 0.28 & 0.28 & $1)$ \\
\hline
\end{tabular}

Figure 3 transitive opening B of the fuzzy proximity R, and its binary weighted tree (with the same shape that the T-transitive closure binary tree, but different values)

\section{Conclusions}

A method to get the transitive closure, a transitive opening and a transitive approximation of a reflexive and symmetric fuzzy relation at the same time is given.

The binary partition trees of the output similarities are the same.

Some examples are provided.

\section{Acknowledgements}

The author would like to thank the anonymous referees for the constructive comments and contributions.

Research partially supported by DGICYT projects number TIN2006-14311 and TIN2006-06190. 


\section{References}

1. Bandler, W. Kohout, J. Special properties, closures and interiors of crisp and fuzzy relations. Fuzzy Sets and Systems 26 (1988) 317-331.

2. De Baets, B., De Meyer, H., Transitive approximation of fuzzy relations by alternating closures and openings, Soft Computing 7 (2003) 210-219.

3. Elorza, J., Burillo, P, On the relation of fuzzy preorders and fuzzy consequence operators, International Journal of Uncertainty, Fuzziness and Knoledge-based Systems 7 (3) (1999).

4. Esteva, F, Garcia, P., Godo, L., Rodriguez, R. O., Fuzzy approximation relations, modal structures and possibilistic logic, Mathware and Soft Computing 5 (2-3) (1998) 151-166.

5. Garmendia, L., Campo, C., Cubillo, S., Salvador, A. A Method to Make Some Fuzzy Relations T-Transitive. International Journal of Intelligence Systems. Vol. 14, No 9, (1999) $873-882$.

6. Garmendia, L, Recasens, J. Finding close T-indistinguishability Operators to a given Proximity. Proceedings EUSFLAT2007. Ostrava. (2007). Vol 1, 127-133.

7. Jacas, J., Recasens, J., Fuzzy T-transitive relations: eigenvectors and generators, Fuzzy Sets and Systems 72 (1995) 147-154.

8. Jacas, J., Recasens, J., Decomposable indistinguishability operators, Proceedings of the Sixth IFSA Congress, Sao Paulo (1995).

9. Larsen H., R. Yager, "Efficient computation of transitive closures," Fuzzy Sets Syst., vol. 38 (1990) 81-90.

10. Lee, H.-S. An optimal algorithm for computing the max-min transitive closure of a fuzzy similarity matrix , Fuzzy Sets and Systems 123 (2001) 129-136.

11. Naessens, H., De Meyer, H., De Baets, B., Algorithms for the Computation of T-Transitive Closures, IEEE Trans Fuzzy Systems 10:4 (2002) 541-551.

12. Ovchinnikov, S. Representations of Transitive Fuzzy Relations, in Aspects of Vagueness, H. J. Skala, S. Termini y E. Trillas (Eds.), Reidel Pubs. (1984) 105-118.

13. Schweizer, B., Sklar A. Probabilistic Metric Spaces, North-Holland, New York, (1984).

14. Trillas, E., Valverde, L., An inquiry into indistinguishability operators, in Aspects of Vagueness, H. J. Skala, S. Termini y E. Trillas (Eds.), Reidel Pubs. (1984) 231-256.

15. Valverde, L., On the structure of F-indistinguishability operators, Fuzzy Sets and Systems 17 (1985) 313-328.

16. Zadeh, L. A., Similarity relations and fuzzy orderings, Inform. Sci. 3 (1971) 177-200. 